\title{
ORIGINAL
}

\section{POSIBILIDADES DE LOS GRUPOS CLÍNICOS AJUSTADOS (AJUSTED CLINICAL GROUPS-ACGS) EN EL AJUSTE DE RIESGOS DE PAGO CAPITATIVO}

\author{
Antoni Sicras-Mainar (1), Josep Serrat-Tarrés (2), Ruth Navarro-Artieda (1) y Josep Ramón Llopart- \\ López (1) \\ (1) Dirección de Planificación y Desarrollo Organizativo. Badalona. Serveis Assistencials. Badalona. Barcelona. \\ (2) Dirección de Salud Pública. Badalona. Serveis Assistencials. Badalona. Barcelona.
}

\section{RESUMEN}

Fundamento: La mayoría de los sistemas de clasificación de pacientes han sido diseñados en Estados Unidos con la finalidad de disponer de una herramienta que facilite una medida en la utilización de los recursos. El objeto del estudio fue obtener los pesos relativos (PR) medios del coste de la asistencia en varios equipos de atención primaria de salud, en comparación con los americanos, mediante la aplicación de los Grupos Clínicos Ajustados (ACGs), como un posible ajuste de riesgos de pago capitativo.

Métodos: Estudio descriptivo. Se incluyeron todas las historias clínicas generadas por cuatro equipos de atención primaria durante el año 2003. Las principales mediciones fueron: edad y sexo, recursos (visitas y costes) y casuística. El modelo de costes para cada paciente se estableció diferenciando los costes fijos y variables. Se efectuó un análisis de regresión para la corrección del modelo. El coste relativo de cada ACG se obtuvo dividiendo el coste medio de cada categoría entre el coste medio de toda la población de referencia.

Resultados: El número total de historias estudiadas fue de 62.311 , con una media de $4,8 \pm 3,2$ diagnósticos y $7,8 \pm 7,5$ visitas/paciente/año. El gasto total fue de 24,1 millones de euros, los costes fijos o semifijos representan el $28,9 \%$ y los variables el $71,1 \%$. El promedio de coste total por paciente/año fue de $387,34 \pm 145,87 €$ (referencia). El poder explicativo corregido del coste de la asistencia entre las dos clasificaciones (estadounidense respecto a la estudiada) fue $\operatorname{del} 64,3 \% ; \mathrm{p}=0,000$ ).

Conclusiones: La generalización de los resultados debe de interpretarse con prudencia. Los ACGs se muestran como un instrumento adecuado y podrían utilizarse los $\mathrm{PR}$ medios estadounidenses para el ajuste del riesgo en el pago capitativo, ante la dificultad de tener bases de datos amplias y consistentes en nuestro medio. Sería necesario esperar nuevas investigaciones que refuercen la consistencia de los resultados.

Palabras clave: Grupos clínicos ajustdos. Economía en Atención de Salud y Organizaciones. Recursos en salud.

Correspondencia:

Antoni Sicras Mainar

C. Gaietà Soler, 6-8 entlo . 08911 Badalona. Barcelona.

Teléfono: 935072684

Correo electrónico: asicras@bsa.gs
ABSTRACT

\section{Prospects of Adjusted Clinical groups (ACG's) in Capitated Payment Risk Adjustment}

Background: Most patient classification systems have been designed in the United States for the purpose of availing of a tool providing a means of gauging the use of resources. This study was aimed at calculating the mean relative weights (MRW's) for the cost of care at several primary care health facilities as compared to those in the U.S. by using the Adjusted Clinical Groups (ACG's) as a possible capitated payment risk adjustment.

Methods: Descriptive study. All of the clinical records generated by four primary care facilities throughout 2003 were included. The main measurements were: age and gender, resources (visits and costs) and casuistics. The cost model was determined for each individual patient by differentiating the fixed and variable costs. A regression analysis was made for model adjustment purposes. The relative cost of each ACG was calculated by dividing the mean cost of each category by the mean cost of the population as a whole.

Results: A total of 62,311 records were studied, revealing an average of $4.8 \pm 3.2$ diagnoses and $7.8 \pm 7.5$ visits/patient/year. The total expense was 24.1 million euros, the fixed and semi-fixed costs totaling $28.9 \%$ and the variable costs $71.1 \%$. The mean total cost/patient/year was $387.34 \pm 145.87$ ? (reference). The adjusted explicative power of the cost of care between the two classifications (U.S. classification vs. the one studied) was $64.3 \%$; $\mathrm{p}=0,000$ ).

Conclusions: The generalization of the results must be carefully construed. ACG's show themselves to be a suitable tool, and the mean U.S. RW's could be used for adjusting capitated payment risk adjustments in view of the difficulty of availing of full, consistent databases in our environment. Further research would be required to back up the consistency of the results.

Key words: Adjusted Clinical Groups. Economics. Health resources. Health Care Economics and Organizations. 


\section{INTRODUCCIÓN}

La mayoría de los sistemas de clasificación de pacientes (SCP) han sido diseñados en Estados Unidos, con la finalidad de disponer de una herramienta que facilitara una medida en la utilización de los recursos hospitalarios ${ }^{1}$. De todos ellos, el único que presenta un elevado poder explicativo sobre los costes de la asistencia en la hospitalización convencional, son los Grupos Relacionados con el Diagnóstico (GRDs) ${ }^{2}$.

Los SCP en atención ambulatoria, y en particular los de la atención primaria, no han sido empleados de una forma generalizada ${ }^{3}$. En este sentido, los Grupos Clínicos Ajustados o Ajusted Clinical Groups (ACGs), son un sistema de agrupación que clasifica a las personas según las enfermedades que presentan durante un período de tiempo. Fueron desarrollados por Starfield ${ }^{4}$ y Weiner ${ }^{5}$ (Universidad Johns Hopkins) y su objetivo es medir el grado de enfermedad en poblaciones de pacientes basándose en niveles de comorbilidad por iso-consumo de recursos. Constituyen en la actualidad, junto con los los Hierarchical Coexisting Conditions (HCC) o los Clinical Risk Groups (CRG), una de las posibles metodologías de ajuste del riesgo, que se pueden utilizar para evaluar de una forma más precisa y equitativa, la financiación por parte de la administración (pago capitativo) o valorar la eficiencia en la utilización de los servicios de salud ${ }^{6-7}$.

En actualidad se está mostrando un creciente interés ante la posibilidad de asignar una financiación per cápita ${ }^{8}$, como mecanismo de asignación equitativa de los recursos asistenciales; pero hay incertidumbre sobre la adaptación de estos instrumentos en un ámbito sanitario distinto a aquel en el que han sido diseñados. En general, la evidencia que se tiene en la aplicación de los ACGs en nuestro medio está estudiada ${ }^{9-14}$, aunque se requiere de un mejor conocimiento que refuerce una situación pragmática ${ }^{9}$.
El objeto del estudio fue obtener los pesos relativos (PR) medios del coste de la asistencia en varios equipos de atención primaria de salud, en comparación con los americanos, mediante la aplicación de los ACGs, como un posible ajuste de riesgos de pago capitativo en situación de práctica clínica habitual.

\section{MATERIAL Y MÉTODO}

Se realizó un diseño descriptivo y retrospectivo, a partir de los registros médicos de pacientes seguidos en régimen ambulatorio. La población de estudio estuvo formada por cuatro centros de atención primaria (ABS Apenins-Montigalá, ABS Morera-Pomar, ABS Nova Lloreda y ABS Montgat-Tiana), pertenecientes a los municipios de Badalona, Montgat y Tiana respectivamente (Barcelona), gestionados por Badalona Serveis Assistencials SA; que tiene asignada a su zona de influencia una población de unos 81.235 habitantes, de la que un $14,1 \%$ son mayores de 64 años. El tipo de organización de los equipos es de carácter reformado, con gestión de titularidad pública y provisión de servicios privada, concertados con el Servicio Catalán de la Salud (CatSalut). Además, la empresa cuenta con una dotación de personal, política de formación, modelo organizativo y cartera de servicios similar a la de la mayoría de centros de atención primaria de Cataluña; con un modelo de gestión descentralizada y servicios estructurales únicos. Fueron incluidos en el estudio todos los pacientes atendidos (asignados o desplazados) por el equipo durante el año 2.003, y, se excluyeron los sujetos trasladados o fallecidos durante el período de estudio.

Las variables de estudio fueron: a) generales: edad y sexo, b) de recursos: visitas realizadas y costes de la asistencia, y c) de casuística o comorbilidad. Se utilizó la variable edad para agrupar a los pacientes: de 0 a 14 años (Pediatría), y los mayores de 14 años (Medicina de Familia). La visita realizada se definió como un contacto entre el equipo de 
profesionales y el paciente por una demanda o problema de salud, en el propio centro o en su domicilio. Fueron excluidas del estudio aquellas visitas realizadas por los especialistas integrados en los propios centros. Se definió el episodio, como un proceso de atención de una enfermedad o una demanda explícita realizada por el paciente. Se consideró equivalente al diagnóstico o motivo de consulta y fueron cuantificados según la Clasificación Internacional de la Atención Primaria (CIAP $)^{15}$. Los episodios seleccionados, no repetidos, en población atendida, se contabilizaron por las fechas de registro en el curso clínico de las historias para cada episodio/motivo de consulta. Asímismo, se realizó una conversión de la CIAP a la Clasificación Internacional de Enfermedades (CIE-9-MC); para su confección se constituyó un grupo de trabajo formado por cinco profesionales (un documentalista, dos médicos clínicos y dos técnicos consultores).

El esquema propuesto para el cálculo de los costes parciales se definió teniendo en cuenta las características de la organización y el grado de desarrollo de los sistemas de información disponibles. La unidad de producto asistencial que sirvió de base al cálculo final, fue el coste por paciente atendido durante el período de estudio. La conciliación de los gastos de la cuenta de pérdidas y ganancias de la Contabilidad Financiera, a los costes de la Contabilidad Analítica, se realizó mediante la conversión de los gastos por naturaleza en costes, y luego a la asignación y clasificación final de los costes. Se excluyeron los gastos de la cuenta de resultados que no se relacionaron directamente con el proceso productivo asistencial (gastos financieros, pérdidas procedentes del inmovilizado, gastos excepcionales y de provisiones procedentes de ejercicios diferentes al periodo analizado. Se definieron como costes por naturaleza las partidas contables pertenecientes a gastos: personal (sueldos y salarios, indemnizaciones y seguridad social a cuenta de la empresa), bienes de consumo (medicamentos, productos intermedios, material sanitario, instrumental; contabilizándose como coste las compras menos la variación de las existencias), y un conjunto de gastos pertenecientes a los servicios externos (limpieza, lavandería), de estructura (reparación y conservación del edificio, vestuario, material de oficina), y de gestión del centro (amortizaciones y tributos). La periodificación de los gastos se realizó en el momento de devengo al finalizar el ejercicio, y para el cálculo de las amortizaciones del inmovilizado, se consideraron desde la adquisición del equipo durante la vida útil del bien, en una depreciación constante anual, de acuerdo con el Plan General Contable para los Centros de Asistencia Sanitaria. Se procedió a la asignación y clasificación de los costes según su dependencia con el volumen de actividad desarrollada en los centros como: costes fijos o semifijos (criterio de imputación: costes indirectos) y costes variables (criterio de imputación: costes directos). Se consideraron como costes directos los relacionados con las solicitudes diagnósticas, terapéuticas o derivaciones efectuadas por los profesionales. Los diferentes conceptos de estudio y su valoración económica fueron los siguientes: análisis clínicos (gasto medio por petición), radiología convencional (tarifa por cada prueba solicitada), transporte sanitario (gasto medio por petición), pruebas complementarias (tarifa por prueba solicitada), interconsultas (derivaciones efectuadas a los especialistas de referencia o a los centros hospitalarios de carácter ordinario o urgentes; tarifa adaptada por derivación), prescripciones (recetas a cargo del CatSalut; precio de venta al público). Las tarifas utilizadas procedieron de estudios de contabilidad analítica realizados en la propia organización, facturas de productos intermedios emitidas por los diferentes preveedores o de precios establecidos por el SCS ${ }^{16-17}$. Los costes por naturaleza de personal, bienes de consumo, servicios externos de estructura y gestión se consideraron como costes fijos o semifijos (criterio de imputación: costes indirectos). Se obtuvo un coste medio por visita realizada y se efectuó un 
reparto directo final para cada paciente. Por tanto, el coste por paciente $(\mathrm{Cp})$, en función del servicio final asignado fue: $\mathrm{Cp}=($ coste medio por visita $*$ número de visitas [costes indirectos] $)+($ costes variables [costes directos]). El coste relativo de cada ACGs se obtuvo dividiendo el coste medio de cada categoría entre el coste medio de toda la población de referencia. De esta forma se obtuvo PR de cada grupo respecto al coste medio total.

El algoritmo de funcionamiento del Grouper ACGs versión 6.0 (que incluye la ponderación de los PR medios americanos para cada uno de los 106 agrupaciones), está formado por una serie de pasos consecutivos hasta la asignación de cada paciente a uno de los grupos. Para la construcción de un ACGs se requiere la edad, el sexo y los episodios codificados según la CIE-9MC. El proceso de CIE-9-MC en ACGs consta de 4 etapas, las dos primeras con el propósito de agrupar una serie de condiciones por similar consumo de recursos y las dos últimas con el objetivo de combinar las agrupaciones más frecuentes. La primera etapa agrupa los diagnósticos de la CIE-9MC a 34 ADGs o Ambulatory Diagnostic Groups (un paciente puede tener uno o más ADG); la segunda a 12 CADGs o Collapsed Ambulatory Diagnostic Groups; la tercera a 25 MACs o Major Ambulatory Categories; y la cuarta a ACGs, por lo que cada paciente es asignado a un solo grupo mutuamente excluyente de iso-consumo de recursos.

Los datos se obtuvieron de forma informatizada, respetándose la confidencialidad de los registros marcada por la ley. La relación de variables cuantitativas se efectuó con la correlación lineal de Pearson y/o el análisis de la varianza. Se efectuó un análisis de regresión múltiple para la corrección del modelo (por mínimos cuadrados), teniendo en cuenta que fueron excluidos del estudio los grupos de la clasificación ACG inferiores a 30 pacientes. El análisis estadístico se estableció con un nivel de significación del 5\%, mediante el aplicativo SPSSW 9.0.

Tabla 1

Características generales de la serie estudiada

\begin{tabular}{|lc|}
\hline \multicolumn{1}{|c|}{ Características generales } & Total \\
\hline Número de habitantes adscritos & 81.235 \\
Número de pacientes atendidos & 62.311 \\
Número de episodios atendidos & 297.750 \\
Número de visitas realizadas & 486.413 \\
\hline Edad media (DE) & $37,2(22,5)$ \\
Sexo (\% mujeres) & 51,6 \\
Promedio de episodios por paciente (DE) & $4,8(3,2)$ \\
Promedio de visitas por paciente (DE) & $7,8(7,5)$ \\
Promedio de episodios por visita & 0,61 \\
Intensidad de uso (\%) & 76,7 \\
\hline
\end{tabular}

DE: desviación estándar 


\section{RESULTADOS}

El número de habitantes adscritos en los cuatro centros fue de 81.235 (Medicina de Familia: 84,7\%; Pediatría: 15,3\%. El número total de pacientes estudiados fue de 62.311, con una media de $4,8 \pm 3,2$ episodios y $7,8 \pm 7,5$ visitas realizadas por paciente/año. La edad media de las mujeres, $38,2 \pm 21,6$ años, fue superior a la de los hombres, $36,8 \pm 24,7$ años; $p=0,000$. En la tabla 1 se detallan las características generales de la serie estudiada, así como algunos indicadores unitarios. La frecuentación fue de 6,0 visitas/habitante/año, siendo superior en el servicio de pediatría 9,6 visitas/habitante/ año.

El gasto total fue de 24,1 millones de euros, los costes fijos o semifijos representan el $28,9 \%$ (sueldos y salarios: $20,1 \%$; bienes de consumo: $4,4 \%$; servicios externos y de estructura: $4,4 \%$ ) y los variables el $71,1 \%$ sobre el total, (prescripción farmacéutica: $65,2 \%$; interconsultas: $2,9 \%$, análisis clínicos: $1,9 \%$; radiología y pruebas:
$1,1 \%)$. El coste unitario total por visita realizada/año fue de 49,62 $\pm 24,71 €$ y el promedio de coste total por paciente/año de $387,34 \pm 145,87$ ? , que fueron considerados como los PR medios de referencia.

La carga de morbilidad atendida fue de 297.750 episodios. Cabe destacar que en 28 categorías ACGs (de las 106 posibles, tabla 2) no se agrupó a ningún paciente (el estudio se realizó con población atendida, no adscrita); no se detectaron errores de codificación y todos los pacientes atendidos se incluyeron en alguna categoría. El 89,5\% de la casuística atendida se agrupa 30 ACGs, el 77,9\% en 20 y el 52,6 en 10. En 34 categorías de la clasificación se agrupan 23.308 pacientes (37,4 $\%$ ) con un PR medio del coste superior a 1; mientras que en 44 categorías se agrupan $39.003(62,6 \%)$ con un PR del coste inferior a 1 (promedio del coste total por paciente/ año).

Finalmente, para confeccionar la matriz de correlaciones (tabla 3 ), se excluyeron a 76 pacientes que corresponden a 10 catego-

Figura 1

Correlación y ajuste de los PM americanos y los obtenidos en atención primaria

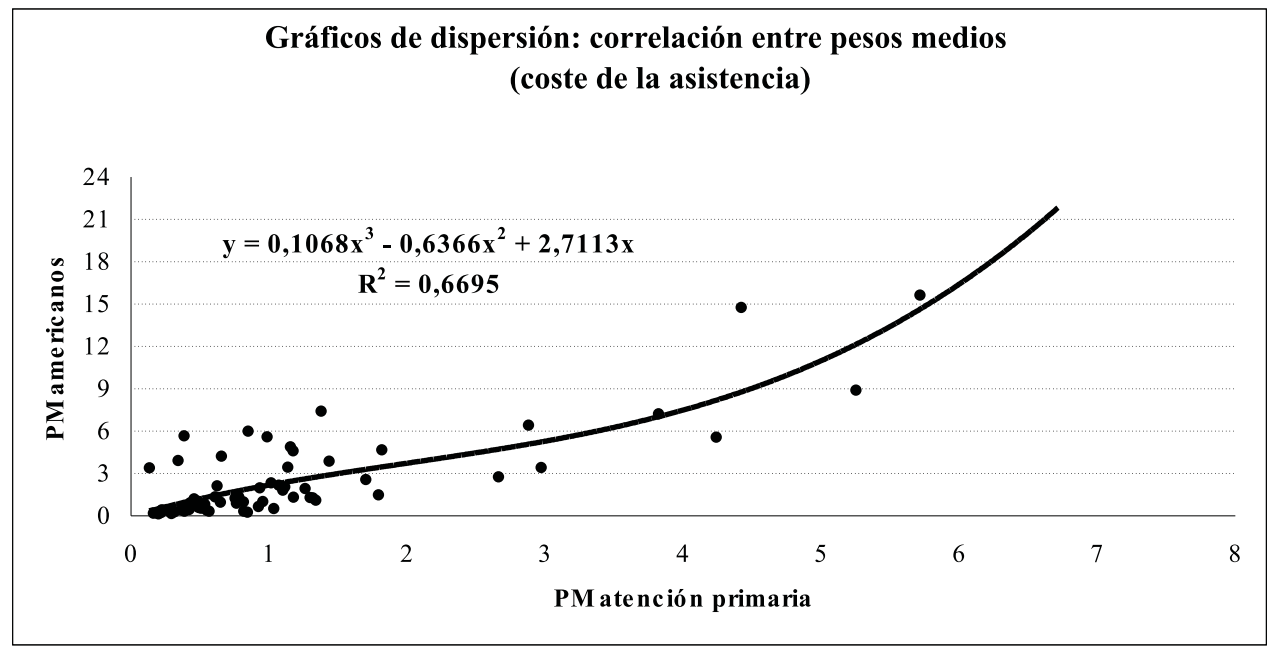

Corrección: regresión polinómica de segundo grado; PM: pesos medios del coste de la asistencia. 
Tabla 2

Descripción de la agrupación de pacientes, visitas y coste de la asistencia en la clasificación ACG (Grupos Clínicos Ajustados)

\begin{tabular}{|c|c|c|c|c|c|c|}
\hline Ord. & . ACG & Descripción & $\begin{array}{c}\text { Casos } \\
\mathbf{N}\end{array}$ & $\begin{array}{c}\text { PR } \\
\text { medio } \\
\text { (visitas) }\end{array}$ & $\begin{array}{c}\text { PR } \\
\text { medio } \\
\text { (coste) }\end{array}$ & $\begin{array}{c}\text { PR } \\
\text { EEUU }\end{array}$ \\
\hline 3 & 0300 & Aguda leve, edad $>=6$ & 8.209 & 0,3582 & 0,1960 & 0,1820 \\
\hline 47 & 4100 & Combinación otros 2-3 ADG, edad $>34$ & 6.537 & 0,9254 & 1,3375 & 1,1110 \\
\hline 66 & 4910 & Combinaciones otros 6-9 ADG, Edad >34, 0-1 ADGs Mayores & 3.954 & 2,2375 & 2,9722 & 3,4320 \\
\hline 27 & 2100 & Aguda leve + recurrente, edad $>5$, sin alergias & 3.921 & 0,6743 & 0,3650 & 0,4270 \\
\hline 53 & 4410 & Combinaciones otros 4-5 ADG, Edad $>44$, sin ADG Mayores & 2.708 & 1,4048 & 1,7934 & 1,4870 \\
\hline 24 & 1800 & Aguda leve + aguda grave & 2.609 & 0,7697 & 0,4091 & 0,7100 \\
\hline 5 & 0500 & Recurrente, sin alergias & 2.461 & 0,3046 & 0,2094 & 0,2450 \\
\hline 54 & 4420 & Combinaciones otros 4-5 ADG, Edad >44, 1 ADG Mayor & 2.332 & 1,6114 & 2,6628 & 2,7590 \\
\hline 4 & 0400 & Aguda grave & 1.899 & 0,3311 & 0,2213 & 0,4160 \\
\hline 16 & 1600 & Preventiva/administrativa & 1.758 & 0,2366 & 0,1985 & 0,1440 \\
\hline 31 & 2500 & Aguda leve + psicosocial, sin trast psiquiátricos graves & 1.634 & 0,6067 & 0,4030 & 0,5820 \\
\hline 38 & 3200 & Aguda leve + aguda grave + recurrente, edad $>11$, sin alergia & 1.592 & 0,9958 & 0,6086 & 1,3550 \\
\hline 42 & 3600 & Aguda leve + aguda grave + recurrente + crónica estable: medicina general & 1.484 & 1,6979 & 1,7000 & 2,5640 \\
\hline 41 & 3500 & Aguda leve + recurrente + psicosocial & 1.421 & 1,0570 & 0,6483 & 0,9700 \\
\hline 29 & 2300 & Aguda leve + crónica estable, medicina general & 1.409 & 0,8792 & 1,0329 & 0,5280 \\
\hline 9 & 0900 & Crónica estable, medicina general & 1.287 & 0,5223 & 0,8157 & 0,3340 \\
\hline 50 & 4310 & Combinaciones otros 4-5 ADG, Edad 18-44, sin ADG Mayores & 1.269 & 1,0517 & 0,7548 & 1,2630 \\
\hline 43 & 3700 & Aguda leve + aguda grave + recurrente + psicosocial & 1.094 & 1,5219 & 1,0702 & 2,1640 \\
\hline 26 & 2000 & Aguda leve + recurrente, edad 2-5 & 971 & 1,4012 & 0,5482 & 0,4010 \\
\hline 67 & 4920 & Combinaciones otros 6-9 ADG, Edad >34, 2 ADGs Mayores & 925 & 2,5073 & 3,8220 & 7,2310 \\
\hline 46 & 4000 & Combinación otros 2-3 ADG, mujeres, edad 18-34 & 910 & 0,6448 & 0,5143 & 0,8510 \\
\hline 45 & 3900 & Combinación otros 2-3 ADG, hombres, edad 18-34 & 835 & 0,6175 & 0,4821 & 1,0280 \\
\hline 34 & 2800 & Aguda grave + recurrente & 813 & 0,5968 & 0,4277 & 0,9120 \\
\hline 2 & 0200 & Aguda leve, edad 2-5 & 734 & 0,7798 & 0,2921 & 0,1740 \\
\hline 13 & 1300 & Psicosocial sin trastornos psiquiátricos graves & 712 & 0,3434 & 0,4209 & 0,4440 \\
\hline 51 & 4320 & Combinaciones otros 4-5 ADG, Edad 18-44, 1 ADG Mayor & 689 & 1,1696 & 1,0150 & 2,3490 \\
\hline 44 & 3800 & Combinación otros 2-3 ADG, edad $<18$ & 559 & 0,9429 & 0,4761 & 0,6870 \\
\hline 76 & 5310 & Neonatos: $0-5$ ADGs no graves & 510 & 1,4643 & 0,4566 & 1,1970 \\
\hline 28 & 2200 & Aguda leve + recurrente, edad $>5$, con alergias & 506 & 0,9366 & 0,5068 & 0,5410 \\
\hline 48 & 4210 & Combinaciones otros 4-5 ADG, Edad 1-17, sin ADG Mayores & 495 & 1,8025 & 0,8133 & 0,9950 \\
\hline 55 & 4430 & Combinaciones otros 4-5 ADG, Edad $>44,+2$ ADGs Mayores & 479 & 1,6952 & 2,8787 & 6,4330 \\
\hline 8 & 0800 & Crónica inestable, medicina general & 385 & 0,4879 & 0,9536 & 1,0230 \\
\hline 36 & 3000 & Aguda leve + aguda grave + recurrente, edad 2-5 & 383 & 1,9796 & 0,7636 & 0,8940 \\
\hline 18 & 1720 & Embarazo: 2-3 ADGs, Sin ADGs Mayores & 349 & 0,6030 & 0,3414 & 3,9220 \\
\hline 37 & 3100 & Aguda leve + aguda grave + recurrente, edad 6-11 & 338 & 1,2608 & 0,5329 & 0,8350 \\
\hline 6 & 0600 & Recurrente, con alergias & 278 & 0,4633 & 0,2814 & 0,3000 \\
\hline 72 & 5040 & Combinaciones otros +10 ADG, Edad 18+, 0-1 ADGs Mayores & 277 & 3,4816 & 4,2388 & 5,5830 \\
\hline 1 & 0100 & Aguda leve, edad $<2$ & 259 & 1,7237 & 0,5649 & 0,3350 \\
\hline 39 & 3300 & Aguda leve + aguda grave + recurrente, edad $>11$, con alergia & 239 & 1,2061 & 0,7812 & 1,3470 \\
\hline 20 & 1740 & Embarazo: 4-5 ADGs, Sin ADGs Mayores & 230 & 1,1433 & 0,6537 & 4,2400 \\
\hline 25 & 1900 & Aguda leve + recurrente, edad $<2$ & 216 & 2,6762 & 0,9225 & 0,6650 \\
\hline 12 & 1200 & Crónica inestable, medicina especializada & 180 & 0,3652 & 0,3858 & 0,3410 \\
\hline 63 & 4810 & Combinaciones otros 6-9 ADG, Mujer, Edad 18-34, sin ADG Mayores & 177 & 1,6677 & 1,1143 & 2,0450 \\
\hline 73 & 5050 & Combinaciones otros +10 ADG, Edad 18+, 2 ADGs Mayores & 152 & 3,9491 & 5,2518 & 8,9150 \\
\hline 68 & 4930 & Combinaciones otros 6-9 ADG, Edad $>34,3$ ADGs Mayores & 146 & 3,0948 & 4,4211 & 14,7740 \\
\hline 49 & 4220 & Combinaciones otros 4-5 ADG, Edad 1-17, +1 ADGs Mayor & 144 & 1,5167 & 0,6227 & 2,1370 \\
\hline 30 & 2400 & Aguda leve + oftalmológica/dental & 142 & 0,6556 & 0,3134 & 0,2780 \\
\hline 11 & 1100 & Oftalmología/dental & 140 & 0,2926 & 0,1626 & 0,2000 \\
\hline 7 & 0700 & Asma & 122 & 0,4294 & 0,8426 & 0,2500 \\
\hline 64 & 4820 & Combinaciones otros 6-9 ADG, Mujer, Edad 18-34, 1 ADG Mayor & 107 & 1,6740 & 1,1356 & 3,4560 \\
\hline 17 & 1710 & Embarazo: 0-1 ADGs & 105 & 0,2654 & 0,1323 & 3,3900 \\
\hline 40 & 3400 & Aguda leve + recurrente + oftalmológica $/$ dental & 99 & 1,0376 & 0,4914 & 0,5820 \\
\hline 56 & 4510 & Combinaciones otros 6-9 ADG, Edad 1-5, sin ADG Mayores & 97 & 3,3371 & 1,2597 & 1,9350 \\
\hline 58 & 4610 & Combinaciones otros 6-9 ADG, Edad 6-17, sin ADG Mayores & 88 & 2,2441 & 1,0981 & 1,8180 \\
\hline
\end{tabular}


Tabla 2 (Continuación)

\begin{tabular}{|c|c|c|c|c|c|c|}
\hline Ord. & . ACG & Descripción & $\begin{array}{c}\text { Casos } \\
\mathbf{N}\end{array}$ & $\begin{array}{c}\text { PR } \\
\text { medio } \\
\text { (visitas) }\end{array}$ & $\begin{array}{c}\text { PR } \\
\text { medio } \\
\text { (coste) }\end{array}$ & $\begin{array}{c}\text { PR } \\
\text { EEUU }\end{array}$ \\
\hline 52 & 4330 & Combinaciones otros 4-5 ADG, Edad 18-44, +2 ADGs Mayores & 83 & 1,1525 & 1,1546 & 4,8920 \\
\hline 35 & 2900 & Aguda leve + aguda grave + recurrente, edad $<2$ & 78 & 3,4273 & 1,1768 & 1,3260 \\
\hline 14 & 1400 & Psicosocial con trastornos psiquiátricos graves, sin leves & 77 & 0,4736 & 1,2977 & 1,2910 \\
\hline 22 & 1760 & Embarazo: 6+ ADGs, Sin ADGs Mayores & 74 & 1,6970 & 1,1736 & 4,6160 \\
\hline 10 & 1000 & Crónica estable, medicina especializada & 72 & 0,3156 & 0,2164 & 0,2460 \\
\hline 32 & 2600 & Aguda leve + psicosocial, con trast psiquiátricos graves, sin leves & 70 & 0,8016 & 1,3176 & 1,2820 \\
\hline 59 & 4620 & Combinaciones otros 6-9 ADG, Edad 6-17, +1 ADGs Mayor & 59 & 2,1763 & 0,9852 & 5,5950 \\
\hline 60 & 4710 & Combinaciones otros 6-9 ADG, Varón, Edad 18-34, sin ADG Mayores & 58 & 1,7157 & 0,9324 & 1,9840 \\
\hline 61 & 4720 & Combinaciones otros 6-9 ADG, Varón, Edad 18-34, 1 ADG Mayor & 55 & 1,7649 & 1,4341 & 3,8800 \\
\hline 21 & 1750 & Embarazo: 4-5 ADGs, +1 ADGs Mayor & 52 & 1,2272 & 0,8474 & 5,9970 \\
\hline 74 & 5060 & Combinaciones otros $+10 \mathrm{ADG}$, Edad 18+, 3 ADGs Mayores & 51 & 4,6425 & 5,7146 & 15,6330 \\
\hline 23 & 1770 & Embarazo: $6+$ ADGs, +1 ADGs Mayor & 38 & 1,8922 & 1,3775 & 7,4110 \\
\hline 57 & 4520 & Combinaciones otros 6-9 ADG, Edad 1-5, +1 ADGs Mayor & 37 & 3,5154 & 1,8159 & 4,6840 \\
\hline 19 & 1730 & Embarazo: 2-3 ADGs, +1 ADGs Mayor & 34 & 0,9403 & 0,3843 & 5,6670 \\
\hline 65 & 4830 & Combinaciones otros 6-9 ADG, Mujer, Edad 18-34, +2 ADGs Mayores & 28 & 2,1965 & 1,5284 & 7,0240 \\
\hline 62 & 4730 & Combinaciones otros 6-9 ADG, Varón, Edad 18-34, +2 ADGs Mayores & 20 & 2,2662 & 1,9552 & 9,5070 \\
\hline 33 & 2700 & Aguda leve + psicosocial, con trast psiquiátricos graves, y leves & 12 & 1,1770 & 1,2787 & 2,4270 \\
\hline 75 & 5070 & Combinaciones otros $+10 \mathrm{ADG}$, Edad $18+,+4$ ADGs Mayores & 10 & 4,4426 & 5,2948 & 35,8000 \\
\hline 69 & 4940 & Combinaciones otros 6-9 ADG, Edad $>34,+4$ ADGs Mayores & 9 & 3,2100 & 4,0102 & 27,7260 \\
\hline 15 & 1500 & Psicosocial con trastornos psiquiátricos graves y leves & 7 & 0,4402 & 0,2837 & 2,4900 \\
\hline 77 & 5320 & Neonatos: $0-5$ ADGs, 10 más graves & 7 & 2,1278 & 0,6910 & 5,5960 \\
\hline 70 & 5010 & Combinaciones otros +10 ADG, Edad 1-17 sin ADG Mayores & 5 & 3,8263 & 1,4712 & 3,8720 \\
\hline 78 & 5322 & Otras combinaciones & 5 & 3,1073 & 1,0522 & 1,2321 \\
\hline 71 & 5020 & Combinaciones otros +10 ADG, Edad 1-17, 1 ADG Mayor & 1 & 4,8792 & 1,6389 & 7,7390 \\
\hline
\end{tabular}

Ord.: ordenación intrínseca de la clasificación; PR: pesos relativos; N: número de pacientes incluidos en cada grupo.

rías ACG $(\mathrm{n}<30)$. El poder explicativo corregido entre los PR del coste de la asistencia entre las dos clasificaciones (americana respecto a la de la atención primaria estudiada) fue de $64,3 \%$, con un coeficiente de colinealidad inferior a 3 .
La correlación entre los pesos medios del coste de la asistencia por paciente/año atendido se detallan en la figura 1. El mejor ajuste del modelo se consigue mediante una regresión polinómica de segundo grado (coeficiente de determinación: 0,$6695 ; \mathrm{p}=0,000$ ).

Tabla 3

Matriz de correlaciones binarias entre los diferentes pesos relativos medios

\begin{tabular}{|lcc|}
\hline \multicolumn{1}{|c}{ Correlaciones binarias: } & r & p \\
\hline PR EEUU - PR coste total en AP & 0,805 & 0,000 \\
PR EEUU - PR visitas realizadas en AP & 0,669 & 0,000 \\
PR coste total en AP - PM visitas realizadas en AP & 0,776 & 0,000 \\
\hline
\end{tabular}

r: correlación lineal de Pearson; p: grado de significación estadística; AP: atención primaria; PR: pesos relativos medios; PM EEUU: pesos medios del coste total de la asistencia americanos; $n>30$ casos por grupo observado 


\section{DISCUSIÓN}

En nuestro entorno, en la mayoría de los centros de atención primaria urbanos y reformados debe de existir una similitud en los estilos de práctica clínica y de protocolarización, que repercuta en una disminución en la variabilidad de las actuaciones ${ }^{18}$. En este escenario, los distintos métodos innovadores de ajuste para la financiación capitativa deberían de pretender calcular como las características de un individuo predice su gasto sanitario para el año siguiente. Sin embargo, algunos de estos individuos no tendrán gasto sanitario al no ser atendidos, por tanto, cualquier método de clasificación según isoconsumo de recursos, debería de predecir las características generales de los pacientes no atendidos, a pesar de las dificultades que puede representar $^{3,19}$. Por tanto, será de suma importancia potenciar la informatización de los centros y establecer mecanismos de consenso entre los profesionales en la uniformidad de los calidad de $\operatorname{los}_{\text {datos }}{ }^{20} \mathrm{y}$ de selección de los episodios, ante una carencia de decisión política para la obtención de un conjunto básico ${ }^{21}$ de datos en atención primaria y/o ambulatoria.

Para cualquier reforma que pretenda un sistema de financiación en grupos poblacionales con cobertura sanitaria, estamos necesitados de algún tipo de instrumento de medida que ajuste el riesgo poblacional ${ }^{1,3,8}$. A pesar de que el poder explicativo de los ajustes regresionales alcanza unos niveles moderados de varianza explicable, están muy por encima de los ajustes convencionales por edad y sexo, al tener en cuenta la carga de morbilidad ${ }^{3,8,22}$. Este modelo debería pretender: mejorar la equidad de acceso de la población, estimular la creación de sistemas integrados de salud mediante alianzas entre proveedores del territorio y mejorar la eficiencia del sistema de salud. La evaluación parcial de los objetivos de este sistema de compra sugiere mejoras en la validez de la fórmula de cálculo, la distribución global de los recursos y el traspaso de una cápita glo$\mathrm{bal}^{6}$. En este aspecto, el papel de los ACGs americanos en la financiación, según los resultados observados, podría tener su aplicación desde un efecto de ajuste, hasta el posible establecimiento de tasas específicas de capitación ${ }^{3}$, según la proporción de usuarios de cada categoría (por estandarización indirecta), sin recurrir al cálculo complejo de los costes reales por cada paciente.

Un aspecto práctico de interés observado en nuestro estudio, coincidente con otros autores $^{3-5,23}$, es la posibilidad del sistema ACGs como un instrumento relativamente neutral en cuanto a la medida de la atención sanitaria, puesto que ofrece pocas posibilidades de perversión. Es menos probable, que se modifique innecesariamente la carga de morbilidad de un paciente, que la programación de visitas o procedimientos diagnósti$\cos ^{3,24-25}$. Además, la agrupación de episodios de un nivel similar, constituye ya un primer filtro que dificulta los cambios que son necesarios para variar la asignación de un paciente a un ACGs específico.

El agrupador ACG necesita un número de variables limitado para cada paciente: edad, sexo y diagnósticos (no necesariamente correlativos en el tiempo); esta simplicidad de uso se ajusta a las necesidades de la atención primaria de salud, con un gran volumen de información en el manejo diario, limitación en el tiempo asistencial, coexistencia de profesionales (médico, enfermera, trabajo social, etc.), y reiteración de pacientes a lo

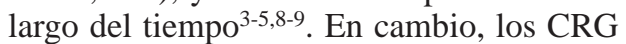
clasifican a los individuos según los niveles de gravedad de su estado de salud y en función de su estado de cronicidad; su ordenación es jerárquica, en primer lugar según el estado de salud (9 grados), en segundo lugar según la categoria de ajuste de riesgo específico (269 tipos) y por último según el nivel de gravedad (1069 agrupaciones en total $)^{7}$. Estos dos sistemas de agrupación de pacientes son los que mejor posicionados están en estos momentos, aunque sería prudente 
esperar nuevas investigaciones que refuercen la consistencia de los resultados.

En este aspecto, y como principales ventajas de los ACGs, como sistema de medida de la carga de morbilidad y de utilización de recursos, podemos citar su sencillez de construcción, manejo pragmático, pocas categorías mutuamente excluyentes y su unidad de medida (paciente). En cambio, su potencial desventaja podría ser la complejidad de comprensión para los clínicos y la inexistencia directa de la severidad de los pacientes atendidos, en comparación con los CRG; siendo el poder explicativo de las dos clasificaciones muy similar ${ }^{4-8}$.

El poder explicativo corregido de los PM del coste de la asistencia entre las dos clasificaciones (americana respecto a la de la atención primaria estudiada) fue de $64,3 \%$. Determinados autores ${ }^{26-28}$ han seguido metodologías de cálculo distintas que incluyen el «coste total de la asistencia», es decir, las visitas efectuadas a los servicios de urgencias, los ingresos hospitalarios y las visitas a especialistas, ya sean derivados por el propio centro o no. Todos ellos coinciden, al igual que en nuestro estudio, que al introducir la morbilidad el poder explicativo aumenta, por tanto es una reflexión interesante y práctica a nivel de gestión clínica, que se deberá de tener presente cuando estemos cuantificando los costes de un equipo. Estas observaciones refuerzan la firmeza de los resultados obtenidos con los diferentes estudios explorados ${ }^{3-5,8-14,22-26}$.

Las limitaciones más destacadas deben de relacionarse con el grado de maduración de los sistemas de información desarrollados en el estudio, la precisión conseguida en la conversión de la CIAP a CIE-9-MC ${ }^{29}$, la exactitud de medida de los costes o la posible variabilidad y/o gravedad ${ }^{30}$ en la selección del episodio de atención por parte de los diferentes profesionales, y sobre todo, en la validez externa de los resultados, que puede ocasionar efectos de contaminación entre los grupos o la existencia de poca especificidad clínica. Por tanto, un posible escenario para la reflexión en la financiación de los equipos de atención primaria, sugiere una combinación mixta: a) ponderación de los costes estructurales vinculados a la accesibilidad, b) presencia de los costes variables en base a la casuística y complejidad de los pacientes, y c) objetivos de calidad derivados de la política sanitaria deseada por el comprador y esperada por el cliente.

En conclusión, los resultados obtenidos deben de interpretarse con prudencia por su validez externa, los ACGs se muestran como un instrumento adecuado y podrían utilizarse los PR medios americanos para el ajuste del riesgo en el pago capitativo, ante la dificultad en nuestro medio, de tener bases de datos amplias y consistentes. Sería necesario esperar nuevas investigaciones que refuercen la consistencia de los resultados.

\section{AGRADECIMIENTOS}

A $\mathrm{M}^{\mathrm{a}}$ A. Cabezas (Gerente), M. Pons (Director Clínico), A. Escosa (Jefe de Atención Primaria) y J. Codes (Director Económico Financiero) de Badalona Serveis Assistencial; a O. Pané y J. Pomar (División de Consultoria y Gestión) del Consorci Hospitalari de Catalunya; y a A. Arias y C. Illa (empresa IASIST). A todos ellos, por el soporte prestado en las diferentes fases del estudio. Y en especial, a los profesionales de atención primaria de Badalona Serveis Assitencials, ya que sin su constante aportación y registro del dia a dia, no hubiera sido posible la realización de este estudio. A las personas encargadas por la revista de evaluar el trabajo, por sus acertados comentarios en la versión anterior de este manuscrito.

\section{BIBLIOGRAFÍA}

1. Petersen LA, Pietz K, Woodard LD, Byrne M. Comparison of the predictive validity of diagnosis- 
based risk adjusters for clinical outcomes. Med Care 2005; 43:61-7.

2. Fetter RG, Shin Y, Freeman JL, Averill RF, Thompson JD. Case mix definition by Diagnosis-Related Groups. Med Care 1980;18(Suppl):1-53.

3. Juncosa S, Bolívar B. Descripció, Comportament, Usos i Metodología d'utilització d'un sistema per mesurar la casuística en la nostra Atenció Primària: els Ambulatory Care Groups. Barcelona: Fundació Salut, Empresa i Economia;1999.

4. Starfield B, Weiner J, Mumford L, Steinwachs D. Ambulatory Care Groups: a categorization of diagnoses for research and management. Health Ser Res 1991;26:53-74.

5. Weiner JP, Starfield BH, Steinwachs DM, Mumford LM. Development and application of a population-oriented measure of ambulatory care casemix. Med Care 1991:29:452-72.

6. Vargas I. La utilización del mecanismo de asignación per cápita: la experiencia de Cataluña. Cuadernos de Gestión 2002;8:167-78.

7. Hughes JS, Averill RF, Eisenhandler J, Goldfield NI, Muldoon J, Neff JM, Gay JC. Clinical Risk Groups (CRGs): a classification system for riskadjusted capitation-based payment and health care management. Med Care 2004; 42:81-90.

8. Madden CW, Mackay BP, Skillman SM, Ciol M, Diehr PK. Risk adjusting capitation: applications in employed and disabled populations. Health Care Manag Sci 2000; 3:101-9.

9. Bolíbar B, Juncosa S. The development of ambulatory care groups in primary care. Rev Neurol 1999;29: 667-669.

10. Bolanos-Carmona V, Ocaña-Riola R, PradosTorres A, Gutierrez-Cuadra P. Variations in health services utilization by primary care patients. Health Serv Manage Res 2002;15:116-25.

11. Carmona G, Prados A, Sánchez-Cantalejo E. Los Grupos de Atención Ambulatoria. Resultados parciales del proyecto: Evaluación del comportamiento de los Grupos de Atención Ambulatoria en nuestro entorno de Atención Primaria. Gestión Hospitalaria 1997;1:40-45.

12. Hormigo A, García AJ, Martos F, García MC, Montesinos AC, Prados A. Evaluación retrospectiva del comportamiento de los grupos de cuidados ambulatorios en un centro de salud. Aten Primaria 1998;21:36-42.
13. Juncosa S, Bolíbar B, Roset M, Tome R. Performance of an ambulatory case mix system in primary care in Spain: Ambulatory Care Groups (ACGs). European J Public Health 1999;9:27-35.

14. Orueta JF, Lopez de Munain J, Baez K, Aiarzaguena JM, Aranguren JI, Pedrero E. Application of the ambulatory care groups in the primary care of a European national health care system: does it work?. Med Care 1999;37:238-248.

15. Lamberts H, Wood M. Clasificación Internacional de la Atención Primaria (CIAP). Clasificación de razones de consulta. Barcelona: Masson/SG;1990.

16. Orden de la Generalitat de Catalunya de 22 de diciembre de 1995. Diari Oficial de la Generalitat de Catalunya 1995; 2148:9689-90.

17. García Cardona F, Molins Pérez G, Farré Pradell J. La contabilidad de costes en atención primaria y cartera de servicios. Aten Primaria 1995; 16:141-5.

18. Sackett D, Rosenberg W, Gray J, Haynes Rb. Richardson WS. Evidence based medicine: what it is and what it isn't. BMJ 1996; 312:71-72.

19. López de Munain J, Grandes G, Orueta JF. Importancia de la calidad de los datos en la validación del case-mix ACG. Aten Primaria 1998; 22:128128.

20. Varela J, Berenguer J, Alonso E, Manzanera R, Picas JM. Los sistemas de información en Atención Primaria. Aten Primaria 1988; 5:543-547.

21. Bolíbar B, Castillo A, Coderch J, Ruano I, Sicras A, Fusté J. Hacia la definición de un conjunto mínimo básico de datos de atención primaria. Aten Primaria 2002;30:229-235.

22. Warner G, Hoenig H, Montez M, Wang F, Rosen A. Evaluating diagnosis-based risk-adjustment methods in a population with spinal cord dysfunction. Arch Phys Med Rehabil 2004; $85: 218-26$

23. Meenan RT, Goodman MJ, Fishman PA, Hornbrook MC, O'Keeffe-Rosetti MC, Bachman DJ. Using risk-adjustment models to identify high-cost risks. Med Care 2003;41:1301-12.

24. Rosen AK, Loveland SA, Rakovski CC, Christiansen CL, Berlowitz DR. Do different case-mix measures affect assessments of provider efficiency? Lessons from the Department of Veterans Affairs. J Ambul Care Manage 2003; $26: 229-42$ 
25. Wahls TL, Barnett MJ, Rosenthal GE. Predicting resource utilization in a veterans health administration primary care population: comparison of methods based on diagnoses and medications. Med Care 2004; 42:123-8.

26. Adams EK, Bronstein JM, Raskind-Hood C. Adjusted clinical groups: predictive accuracy for Medicaid enrollees in three states. Health Care Financ Rev 2002; 24:43-61.

27. Reid RJ, MacWilliam L, Verhulst L, Roos N, Atkinson M. Performance of the ACG case-mix system in two Canadian provinces. Med Care 2001; 39:86-99.
28. Fishman PA, Goodman MJ, Hornbrook MC, Meenan RT, Bachman DJ, O'Keeffe Rosetti MC. Risk adjustment using automated ambulatory pharmacy data: the RxRisk model. Med Care 2003; 41:84-99.

29. Gosálbez Pastor E, Pérez-Salinas I, Díez-Domingo J,Ballester Sanz A, Costa-Alcaraz AM. Sistemas de información en atención primaria: ¿debemos codificar con la CIE-9-MC? Aten Primaria 2003; 31:519-23.

30. Martínez C, Juncosa S, Roset M. ¿Está relacionada la gravedad con la utilización de recursos? Una exploración del Duke Severity of Illness Scale (DUSOI). Aten Primaria 1998; 22:285-92. 